\title{
SoftMax Recurrent Neural Network based Faded Channel Estimation using FIM in MIMO-OFDM
}

\author{
Gulzar Ansari \\ M. Tech. Scholar \\ Department Of ECE, Technocrats Group of Institutions -A \\ Bhopal, India \\ gulzaransari200@gmail.com
}

\author{
Dr. Abhishek Bhatt \\ Professor \\ Department of ECE, Department of ECE, Technocrats \\ Group of Institutions -A, Bhopal \\ India
}

\begin{abstract}
The combination of MIMO technology with OFDM system, there is enhancement of wireless digital communication which is quite beneficial for future communicating system. MIMO-OFDM improves the efficiency and quality of the wireless scenario. With efficient channel estimation technique especially non-blind under MIMO-OFDM scenario present an enhanced performance with low complexity. In the pilot type, the least squares method (LS method) is less complex and requires an implicit knowledge of the channels. However, it suffers from inter-carrier interference (ICI). For this reason, the optimal design of Channel Estimator is an area of ongoing research. In this work, the performance of the DWT-OFDM scheme in combination with a multi-input system and multiple outputs for unknown pilot symbols is evaluated using a neural network. Take advantage of time series prediction using a recurrent neural network (RNN) with a SoftMax layer with frequency index modulation to perform channel prediction. In this research work a comparative analysis is performed among proposed softmax recurrent neural network based channel estimation with existing channel estimation technique for variable signal to noise ratio $(\mathrm{Eb} / \mathrm{No})$ for frequency indexed modulation techniques. The existing channel estimation techniques for MIMO-OFDM communicating environment are based on known pilots over the noisy fading wireless environment. From simulation result, it is observed and concluded that the existing channel estimation techniques gives higher BER as compared to proposed softmax recurrent neural network channel estimation technique.
\end{abstract}

Keywords- MIMO-OFDM, Frequency Index modulation, Inter Carrier Interference, Channel State Information, Channel Estimation, Recurrent Neural network

\section{INTRODUCTION}

In the current communication environment, there is a demand for high speed data transmission and reliable high speed. This requirement represents a paradigmatic challenge for the parallel data transmission system that solves the problems associated with serial systems [1]. The high spectral efficiency and the immunity to interference due to multipath effects are the necessary basis to meet the requirements of today's wireless communication [2].

Orthogonal frequency division multiplexing (OFDM) is a type of block modulation scheme for wireless digital communication technique. With an advancement in wireless technology, higher data bit rate is required that can be achieved by multi-carrier OFDM transmissions. The data transmitted in noisy channel which results in reception of incorrect data bits. At transmitter end error correcting code is added additionally to the data stream for correcting the received incorrect bits. But sometimes, error correcting code not completely recover the original bits.

One of the multi sub carrier modulation scheme is OFDM that is integrated with high data transmission rate, bandwidth efficiency and robust performance in noisy and multipath fading channel. In this technique the available carrier is divided into multiple parallel sub carriers that are orthogonal in nature. Each sub carrier is then modulated separately with different frequency range at different speed and delivers higher efficiency [3].

With the increasing demand requirement of data rates with less noisy bits, the current wireless communication scenario is motivated towards this multicarrier modulation scheme i.e. OFDM. Among all the available multicarrier modulation schemes, OFDM is achieving greater attention from researchers [4].

For improvement of OFDM system performance among all frequency selective channels methodologies, it is required to estimate channel noise as for receiving data bits error free or with less error rate [5]. By estimating noise, it can be found that what effect transmission medium causes on input bits. For this purpose there are many techniques developed that estimate channel noise [6]. Generally, channel estimation techniques are designed as non-blind or semi-blind or blind, out of them non-blind are preferred mostly. But in limited bandwidth scenario, non-blind or pilot-based channel estimation technique will result in wastage of bandwidth. So, in such scenario semi-blind or blind channel estimation technique is preferred more [7].

Some of the pilot-based channel estimation techniques least-squares (LS), minimum mean-square error (MMSE) and linear minimum mean-square error (LMMSE) estimators [2,6]. Many researchers have developed the advancement in these techniques and designed its improved versions because they listed some of the limitations such as:

i. LS channel estimation techniques provide the poor performance and quality of service and gives more mean square error with respect to increasing noise in the channel i.e. signal-to-noise ratios (SNR). At low SNR it gives more error. 
ii. MMSE channel estimation technique is more complex in implementation and are based on channel time statistics. It provide less error as compared to LS channel estimation but MMSE also gives high MSE rate at high SNR values. So, it is also not that efficient.

iii. In LMMSE channel estimation techniques, gives significant performance as compared to LS and MMSE for sample spacing channels but error rate is not that much improved.

So, the performance of LMMSE channel estimation technique are improved on the basis of real time data bits in time domain channel parameters.

Whereas in blind channel estimation techniques, there is no requirement for pilot bits. So, it reduces the channel bandwidth wastage [8]. But it requires some mathematical properties to be calculated for data bits that are sent which makes it more complex as compared to non-blind techniques. Some of types of blind channel estimation techniques are recursive least square (RLS) or LMS. Due to its computational complexity it is not preferred most and are often used in limited bandwidth scenario.

In this research work, neural network is designed for channel estimation technique as an substitute to non-blind channel estimation techniques. As it doesn't need any pilot symbols for estimating noise. Several neural network are designed by researchers for estimation technique. In this research work recurrent backpropagation neural network is

TABLE I

EXISTING CONTRIBUTIONS IN CHANNEL ESTIMATION

\begin{tabular}{|c|c|c|c|c|c|}
\hline Author & Technique Used & SNR (in db) & BER & MSE & RMSE \\
\hline Liu et al. [1] & $\begin{array}{l}\text { Online Extreme Learning } \\
\text { Machine }\end{array}$ & $0-30 \mathrm{db}$ & $10^{-1}$ to $10^{-4}$ & - & - \\
\hline Liu et al. [2] & autoregressive (AR) Model & $0-30 \mathrm{db}$ & - & $10^{-1}$ to $10^{-4}$ & - \\
\hline Yujie Fan et al. [3] & PA-ASSP & $0-30 \mathrm{db}$ & $10^{-1}$ to $10^{-2}$ & $10^{-1}$ to $10^{-4}$ & - \\
\hline \multirow{2}{*}{ Hao Wu et al. [4] } & Extended $\mathrm{H}_{\infty}$ Filter (EHF) & $-30-30 \mathrm{db}$ & - & - & $10^{0}-10^{3}$ \\
\hline & Extended Kalman Filter (EKF) & $-20-20 \mathrm{db}$ & - & - & $10^{0}-10^{4}$ \\
\hline $\begin{array}{l}\text { Aqiel Almamori, } \\
\text { Seshadri Mohan [5] }\end{array}$ & Kalman Filter & $0-40 \mathrm{db}$ & $10^{-2}-10^{-5}$ & - & - \\
\hline \multirow{3}{*}{ Pham et al. [6] } & Kalman & $0-40 \mathrm{db}$ & $10^{-1}-10^{-2}$ & - & - \\
\hline & Extended Kalman Filter (EKF) & $0-40 \mathrm{db}$ & $10^{-1}-10^{-2}$ & - & - \\
\hline & Extended Kalman Filter-CFO & $0-40 \mathrm{db}$ & $10^{-1}-10^{-3}$ & - & - \\
\hline \multirow{4}{*}{$\begin{array}{c}\text { M.Raju and } \\
\text { K.Ashoka Reddy } \\
{[9]}\end{array}$} & MMSE (BPSK) & $0-30 \mathrm{db}$ & - & $\begin{array}{l}\text { Between } 10^{-} \\
2 \text { and } 10^{-3}\end{array}$ & - \\
\hline & LS (BPSK) & $0-30 \mathrm{db}$ & - & $\begin{array}{l}\text { Between } 10^{-} \\
4 \text { and } 10^{-7}\end{array}$ & - \\
\hline & MMSE (8-QAM) & $0-30 \mathrm{db}$ & - & $\begin{array}{l}\text { Between } 10^{-} \\
2 \text { and } 10^{-5}\end{array}$ & - \\
\hline & LS(8-QAM) & $0-30 \mathrm{db}$ & - & $\begin{array}{c}\text { Between } 10^{-} \\
4 \text { and } 10^{-7}\end{array}$ & - \\
\hline \multirow{2}{*}{$\begin{array}{l}\text { Kalpesh Hiray, K. } \\
\text { Vinoth Babu [10] }\end{array}$} & MLP channel estimation & $0-8 \mathrm{db}$ & $10^{-1}-10^{-2}$ & - & - \\
\hline & LMS channel estimation & $0-8 \mathrm{db}$ & $10^{-1}-10^{-4}$ & - & - \\
\hline
\end{tabular}




\begin{tabular}{|c|c|c|c|c|c|}
\hline \multirow{2}{*}{$\begin{array}{c}\text { Yihua Yu and Yuan } \\
\text { Liang [13] }\end{array}$} & Extended Ho Filter (EHF) & $0-18 \mathrm{db}$ & - & - & $\begin{array}{c}10^{-0.8}- \\
10^{-1}\end{array}$ \\
\cline { 2 - 5 } & Extended Kalman Filter (EKF) & $0-18 \mathrm{db}$ & - & - & $\begin{array}{c}10^{-0.8}- \\
10^{-1}\end{array}$ \\
\hline \multirow{2}{*}{$\begin{array}{c}\text { Jun-Han Oh and } \\
\text { Jong-Tae Lim [15] }\end{array}$} & $\begin{array}{c}\text { Two-step channel estimation } \\
\text { (8-pilot) }\end{array}$ & $0-30 \mathrm{db}$ & $10^{0}-10^{-3}$ & - & - \\
\cline { 2 - 5 } & LS estimation (8 pilot) & $0-30 \mathrm{db}$ & $10^{0}-10^{-1}$ & - & - \\
\cline { 2 - 5 } & LS estimation (16-pilot) & $0-30 \mathrm{db}$ & $10^{0}-10^{-2}$ & - & - \\
\hline
\end{tabular}

\section{Proposed Methodology}

The focus of this paper is to carry out that the proposed recurrent neural estimator channel estimation technique for MIMO-OFDM (Orthogonal Frequency Division Modulation) system.

In the wireless communication atmosphere, the signal transmission loss and weakening are the challenging drawback. Orthogonal frequency-division multiplexing (OFDM) is an economical, high data-rate transmission technique for mobile and wireless communication. OFDM take benefits of high-bandwidth potency, and supply straightforward and economical implementation with fast Fourier transform (FFT) and also the Inverse fast Fourier transform (IFFT), reduction of intersymbol interference (ISI) by inserting the cyclic prefix (CP), and strength to multi-path fading and delay [1-3]. The most advantage of OFDM transmission is its strength to frequency selective fading characteristics of a mobile radio channel [6].

In OFDM, the complete signal bandwidth divided into variety of narrow bands or orthogonal subcarriers and it transmit high rate stream of knowledge with single subcarrier, OFDM makes use of an oversized range of closely spaced orthogonal subcarriers that are transmitted in parallel. Therefore, it reduces inter symbol interference (ISI) [4-5].

With the assistance of channel estimation techniques, the noise within the wireless channel is estimated that get additional within the transmitted information and channel equalization technique removes the error, that's estimated from the channel, from the received information and provides the corrected received output to the receiver [8].

Principally the channel is estimated by providing the pilot symbols at the side of the sending signal that is understood at the receiver [9]. The pilot-aided channel estimation is wide used and has additional steady performance. the aim of pilot-aided channel estimation is to get the channel state information (CSI) through the pilot sequence.

The proposed channel estimation will be done using MIMO-OFDM. Firstly, serial data stream is converted into parallel data stream. The data to be transmitted is modulated by applying frequency indexed modulation techniques. After that OFDM with IDWT is applied because it yields the lowest average bit error probability. After that data is transmitted in noisy channel i.e. AWGN and Rayleigh fading channel. The estimation of channel and fading is done using recurrent neural network. Neural network has some outstanding merits such as nonlinear mapping and trainable weights, which means that it can be used to restore nonlinear distortion and learn the information about channels. At the end, BER and
MSE rate will be calculated as performance parameter. Figure 1 shows the proposed flow chart.

The proposed channel estimation algorithm works as follows:

1. Generate the random data input

2. Perform data encoding using convolution encoder

3. Modulate the encoded data

4. Then OFDM transmission is performed using IDWT transform

5. Compute the noise free data (input data), $x$ and channel noise $\mathrm{n}$

6. Receive the noisy data, $\mathrm{y}=\mathrm{x}+\mathrm{n}$

7. Train the network using input data

8. Estimate the channel error on received data using trained neural network and calculate MSE

9. Perform DWT transform on received data and perform demodulation

10. Decode the received data using veterbi decoder

11. Estimate the BER for the OFDM transmission

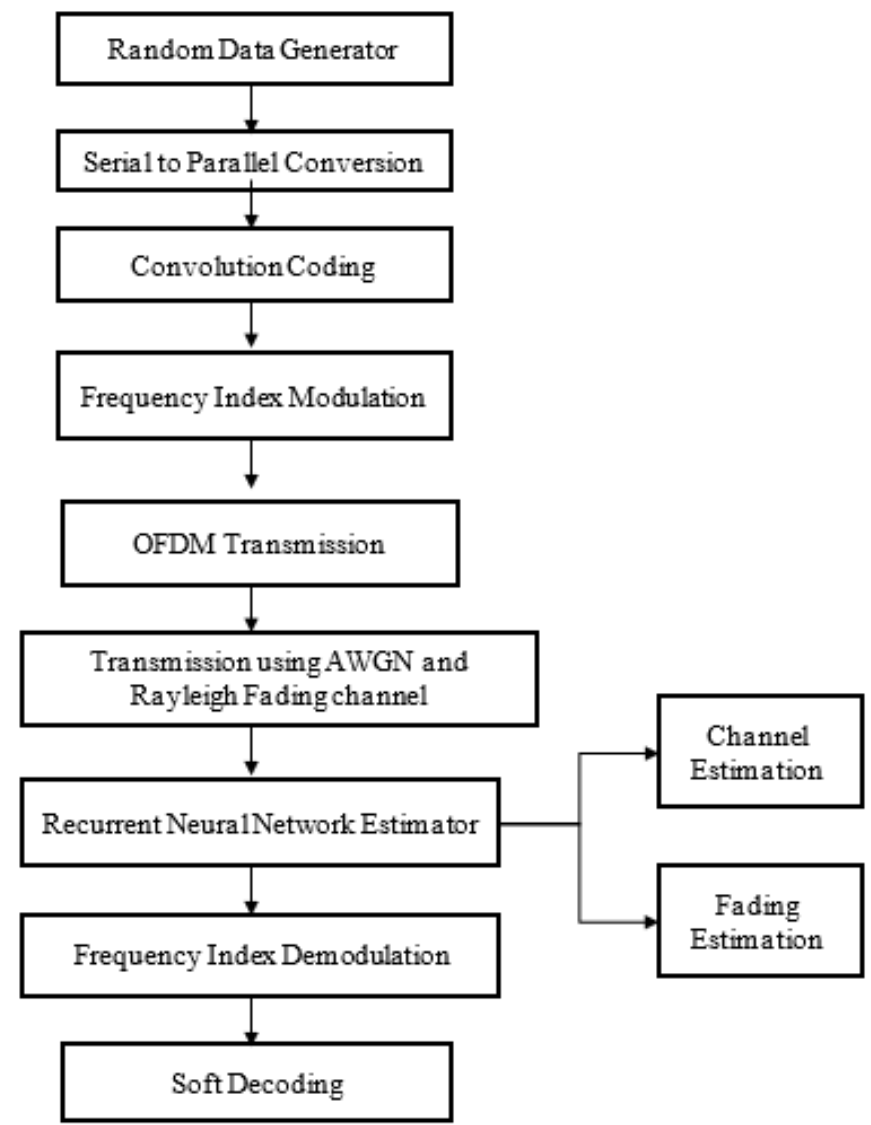

Figure 1: Proposed Work Flowchart 


\section{A. Transmitter End}

The capacity of Random Number Generator is to create irregular bits required for transmission. The convolution codes are different from the square codes in the operational strategy method. A convolutional encoder works on serial information, although the part codes work on a square of information. The use of memory components in convolutional encoders is also unique. The model considered for the implementation of the OFDM transmitter in proposed methodology. The serial to parallel converter receive the $\mathrm{M}$ serial bits to be transmitted, and those bits are divided into $\mathrm{N}$ sub-blocks of mn bits each sub-block. The Inverse Discrete Wavelet Transform (IDWT) transforms the signals from the frequency domain to the time domain; an IDWT converts a number of complex data points, of length that is power of 2, into the same number of points but in the time domain.

Proposed System is designed for wavelet based OFDM under the joint effect of fading \& noise with frequency index modulation. In the proposed system the channel will be modeled by a Rayleigh Fading model followed by AWGN as the additive noise. The equation for this transmission is given below:

$$
x^{\prime}=x+n
$$

Where, $\mathrm{x}$ is transmitting signal and $\mathrm{n}$ is noise added in the channel. The new signal is modified with noise. So, the receiving $\mathrm{M}_{\mathrm{r}}$ will receive $x^{\prime}$.

Finally, the cyclic prefix (CP) of length Lcp, which has to be longer than the channel's delay spread, is concatenated at the front of the symbols for eliminating the effects of the intersymbol-interference over multipath fading channels. Then, the resultant signals are transmitted simultaneously from the $\mathrm{M}$ transmit antennas over the wireless channel.

\section{B. Receiver End}

The demodulation can be made by DWT. At the decoder, a mapped symbol (point) of the transmitted constellation may have changed due to the additive noise in the communications channel, a mis-adjustment in the sampling time at the receiver, or several other unwanted causes.

Using the channel model, at the $n_{\mathrm{c}}$ th subcarrier between the $\mathrm{m}$-th transmit antenna as well as the $\mathrm{n}$-th receive antenna and following the $\mathrm{CP}$ removal and DWT operation.

$$
\mathrm{Y}[\mathrm{n}]=\mathrm{H}[\mathrm{n}] * \mathrm{~S}[\mathrm{n}]+\text { noise }[\mathrm{n}]
$$

Where, $Y[n]=$ Received at $n_{\text {th }}$ sub-carrier.

$\mathrm{H}[\mathrm{n}]=$ Channel Matrix

$\mathrm{S}[\mathrm{n}]=$ Compressed $\mathrm{b}_{\mathrm{g}}$ transmitted bits

Noise $[\mathrm{n}]=$ noise in the channel

The received signal $\mathrm{Y}[\mathrm{n}]$ is then passed through the block splitter and the received symbols in $\mathrm{N}$ groups are detected separately. Then the received signal $\mathrm{Y}[\mathrm{n}]$ in the $\mathrm{n}$-th group before detection can be obtained. As orthogonal frequency division multiplexing (OFDM) supports a high data rate in time delay spread environments with efficient equalization. As equalization requires Channel State Information (CSI), pilots on predetermined subcarriers are sent as training signals for OFDM systems.

In the channel estimation, it is assumed that intersymbol interference is dropped by guard interval, and the received signal is considered as in equation (iii):

$$
\mathrm{Y}(\mathrm{k})=\mathrm{X}(\mathrm{k}) \mathrm{H}(\mathrm{k})+\mathrm{W}(\mathrm{k})
$$

Where $\mathrm{Y}(\mathrm{k})$ is the received signal vector, $\mathrm{X}(\mathrm{k})$ is a matrix containing the transmitted signaling points on its diagonal, $\mathrm{H}(\mathrm{k})$ is a channel attenuation vector, and $\mathrm{W}(\mathrm{k})$ is a noise vector.

According to the adjustment plot, the demodulation is done and the message bits are extracted from the encoded data. A soft choice decoder has been utilized to translate the encoded information. The soft choice decoder Algorithm finds a most maximum likelihood (ML) estimate of a transmitted code arrangement $\mathrm{c}$ from the comparing got grouping $\mathrm{r}$ by augmenting the likelihood $\mathrm{p}(\mathrm{r} \mid \mathrm{c})$ that succession $\mathrm{r}$ is gotten adapted on the evaluated code arrangement c. Grouping c must be a valid coded arrangement. The basic operations which are carried out as per soft choice decoder after receiving one codeword are summarized below:

1. All trellis states are converted into branch metrics.

2. The accumulated metrics of all paths leading to a state are calculated taking into account the "accumulated path metrics" of the states from which the last branches come.

3. Path having at least one minimum 'accumulated path metric' is chosen as the 'survivor path' for the state.

4. So, at the end of this process, each state has one 'survivor path'.

Steps a) to d) are repeated till sufficient number of codewords has been received.

\section{Recurrent Neural Network based Fading Channel Estimation}

The layer of Recurrent Neural Network with SoftMax contains input layer, hidden layer and output layer. The input layer is for passing the input values.

The value at hidden layer is calculated as:

$$
\hat{X}=\sum W * X_{t}+B_{t}
$$

Where, $\mathrm{W}=$ weight matrix

$\mathrm{X}=$ input data values coming from input layers at time instance $t$

$\mathrm{B}=\mathrm{Bias}$ Matrix in the hidden layer

And transfer function is calculated as :

$$
f(x)=\frac{1}{1+e^{-y}}
$$

Where, e=error value

The Softmax Recurrent Neural Network involves the loss function that is to be minimized as :

$$
\begin{aligned}
& \text { loss }_{\min } \\
& =\mid X \\
& -\left(W_{1} \theta\left(W_{2} \theta \ldots \ldots \ldots .\left(W_{l}(f(x))\right)\right)\right) \mid
\end{aligned}
$$

Where, $\mathrm{W}_{1}, \mathrm{~W}_{2}, \ldots \ldots \mathrm{W}_{1}=$ weight function of all hidden layers

$\theta=$ Hidden layers

$\mathrm{f}(\mathrm{x})=$ function to calculate data values at each layer

\section{Softmax classifier}

A frequent use of the softmax function appears in the domain of machine learning, it associate with each output possibility 
a score, which is converted into probability with the softmax function. When a classification task has more than two classes, it is standard to use a softmax output layer. It is the latest layer and it offers a way to predict a discrete probability distribution over the classes.

Observing the MIMO channel model and the structure of a neural network, high similarity that both have multiple inputs and outputs with fully weighted connections can be found. A neural network well suits to process MIMO channels by adapting the number of input and output neurons with respect to the number of transmit and receive antennas. A RNN predictor is quite flexibly to be configured to forecast channel response or envelope on demand in either frequency flat or frequency-selective fading channels. In this chapter, the discussion starts from the simplest case that applies a RNN to predict a flat fading noisy channel in a SISO system, then extends step by step until a frequency-domain predictor for frequency-selective MIMO channels.

\section{RESUlt ANALYSIS}

\section{A. Result Analysis}

In this research work, in MIMO-OFDM system, neuroestimator is proposed for channel estimation mean square error and bit error rate are used as a performance parameter over some of the existing techniques. These are discussed below as:

\section{1) Mean Square Error (MSE)}

Mean square error is the average square of the error of the received bits as compared to the transmitted bits. The error is calculated as the difference between actual values and estimated values.

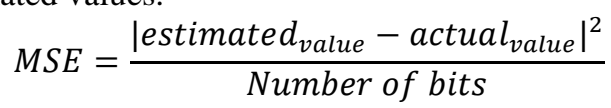

\section{2) Bit Error Rate (BER)}

Bit error rate is termed as error between transmitted bits and received bits in the transmission channel.

$$
B E R=\text { transmitted }_{\text {bits }}-\text { received }_{\text {bits }}
$$

\section{B. Result Analysis}

\section{1) MSE comparison for Channel Estimation}

In this section, the performance of the proposed softmax neural network based channel estimation technique is given under frequency index modulation.

\section{TABLE II}

\begin{tabular}{|c|c|}
\hline \multicolumn{2}{|c|}{ SNR V/S MSE FOR CHANNEL ESTIMATION } \\
\hline SNR & $\begin{array}{c}\text { Softmax Recurrent Neural Network } \\
\text { based Channel Noise Estimation }\end{array}$ \\
\hline 5 & $10^{-6}$ \\
\hline 10 & $10^{-7}$ \\
\hline 15 & $10^{-7}$ \\
\hline 20 & $10^{-7}$ \\
\hline
\end{tabular}

\begin{tabular}{|l|c|}
\hline 25 & $10^{-7}$ \\
\hline 30 & $10^{-7}$ \\
\hline
\end{tabular}

Table II represents the MSE performance of Softmax Recurrent Neural Network based Channel Estimation for FIM modulation. The result is analyzed for SNR varying from 5$30 \mathrm{db}$.

The results in Figure 2 shows that the proposed algorithm performs better than the earlier estimation and equalization model since different training schemes are used in the second and third layers of the softmax RNN. The softmax layer in the output layer of RNN makes the estimates more accurate. The feedback of the estimated parameters in hidden layers of $\mathrm{RNN}$ also enhances the estimation performance.

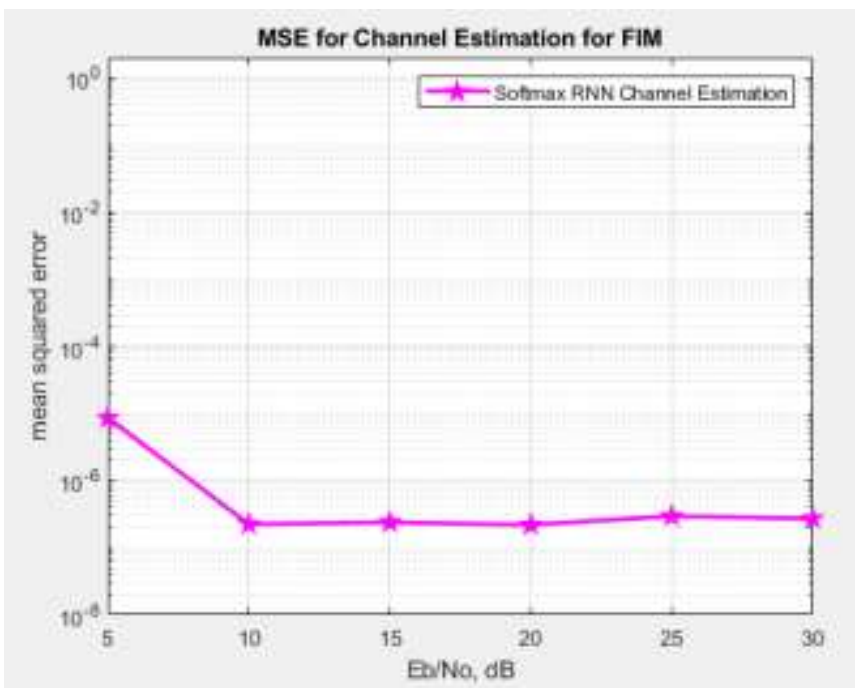

Figure 2: SNR V/s MSE for Channel Estimation

2) BER comparison for Channel Estimation

In this section, the performance of the proposed softmax neural network based channel estimation technique is given under frequency index modulation.

TABLE III

SNR V/S BER FOR CHANNEL ESTIMATION

\begin{tabular}{|c|c|}
\hline SNR & $\begin{array}{c}\text { Softmax Recurrent Neural Network based } \\
\text { Channel Estimation }\end{array}$ \\
\hline 5 & $10^{-1}$ \\
\hline 10 & $10^{-2}$ \\
\hline 15 & $10^{-2}$ \\
\hline 20 & $10^{-4}$ \\
\hline 25 & $10^{-5}$ \\
\hline 30 & $10^{-6}$ \\
\hline
\end{tabular}

Table III represents the BER performance of Softmax Recurrent Neural Network based Channel Estimation for FIM modulation. The result is analyzed for SNR varying from 5$30 \mathrm{db}$. 


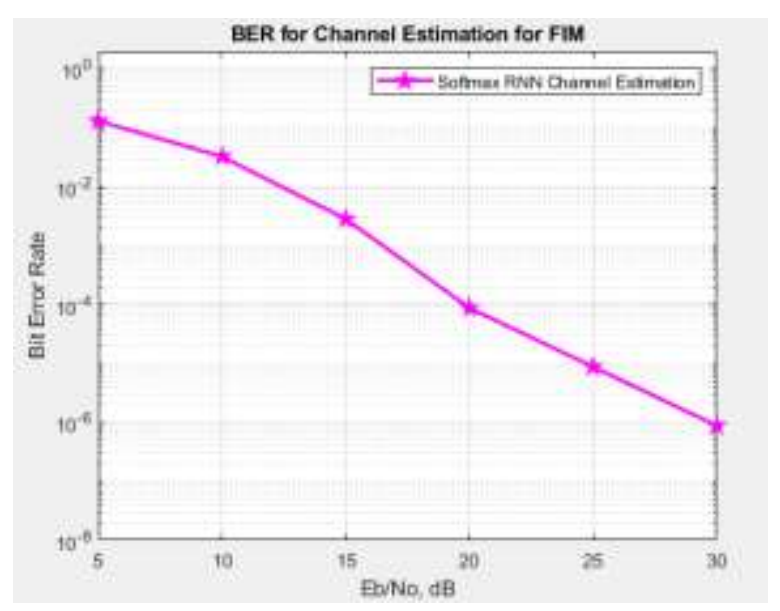

Figure 3: SNR V/s BER for Channel Estimation

The results in Figure 3 shows that the proposed algorithm performs less BER than the earlier estimation and equalization model since different training schemes are used in the second and third layers of the softmax RNN. The softmax layer in the output layer of RNN makes the estimates more accurate. The feedback of the estimated parameters in hidden layers of RNN also enhances the estimation performance.

\section{3) Comparison with Existing Work}

In [1] author proposed an online extreme learning machine (O-ELM)-based channel estimation and equalization scheme with a single hidden layer feedforward network (SLFN) for orthogonal frequency-division multiplexing (OFDM) systems against fading channels and the nonlinear distortion resulting from an high-power amplifier (HPA). Computer simulations show that the proposed scheme can acquire the information of channels accurately and has the ability to resist nonlinear distortion and fading without pre-training and feedback link between receiver and transmitter. Similarly, in [8] proposed Least square (LS) method is considered for sparse channels. Channel coefficients for OFDM are estimated by applying this method and Minimum Mean Square Error (MMSE) method. Results show that the performance of MMSE technique is better than LS method. It is found that the LS method for sparse channel gives lower bit error rate than the general LS method.

The comparative performance of the softmax recurrent neural network-based channel estimation is compared with the Channel Estimation techniques in [1] and [8].

TABLE IV

COMPARATIVE BER PERFORMANCE

\begin{tabular}{|c|c|c|}
\hline Technique & SNR (in db) & BER \\
\hline $\begin{array}{c}\text { Softmax Recurrent Neural } \\
\text { Network based Channel } \\
\text { Estimation }\end{array}$ & $0-30 \mathrm{db}$ & $10^{-1}$ to $10^{-6}$ \\
\hline $\begin{array}{c}\text { Online Extreme Learning } \\
\text { Machine channel estimation [1] }\end{array}$ & $0-30 \mathrm{db}$ & $10^{-1}$ to $10^{-4}$ \\
\hline $\begin{array}{c}\text { Least Square and Minimum } \\
\text { Mean Square Error [8] }\end{array}$ & $0-40 \mathrm{db}$ & $10^{0}$ to $10^{-3}$ \\
\hline
\end{tabular}

TABLE V

PERFORMANCE COMPARISON WITH EXISTING WORK

\begin{tabular}{|c|c|c|c|}
\hline \multirow{2}{*}{ Parameters } & \multicolumn{2}{|c|}{ Previous Results } & $\begin{array}{c}\text { Proposed } \\
\text { Result }\end{array}$ \\
\hline & $\begin{array}{c}\text { Online } \\
\text { Extreme } \\
\text { Learning } \\
\text { Machine } \\
\text { channel } \\
\text { estimation } \\
{[1]}\end{array}$ & $\begin{array}{c}\text { Least } \\
\text { Square and } \\
\text { Minimum } \\
\text { Mean } \\
\text { Square } \\
\text { Error }[8]\end{array}$ & $\begin{array}{c}\text { Space Time } \\
\text { FIM-OFDM } \\
\text { with } \\
\text { Softmax } \\
\text { RNN } \\
\text { channel } \\
\text { estimation }\end{array}$ \\
\hline Performance & Good & Good & better \\
\hline BER & $10^{-1}$ to $10^{-4}$ & $10^{0}$ to $10^{-3}$ & $10^{-2}$ to $10^{-5}$ \\
\hline
\end{tabular}

Table IV and V the tabular illustration of the performance of different parameters discussed in this research work. The proposed work is approx. $2 \%$ improvement of BER compared to previous result. From the analysis of the results, it is found that the proposed work gives a superior performance as compared with the previous result.

\section{V.CONCLUSION}

In wireless communications, multiple signals are transmitted in the channel using the concept of orthogonal frequency division multiplexing (OFDM), which effectively manages the interference between symbols and efficiently uses the available frequency and bandwidth. Since the transmitting antenna transmits the signal in the noisy channel, it is necessary to estimate the noise in the channel. The channel estimation technique in noisy channels allows you to analyze the impact of noise on transmitted data. Orthogonal frequency division multiplexing (OFDM) therefore plays an important role in wireless communication due to its high transmission speed.

Orthogonal Frequency Division Multiplexing (OFDM) based on index modulation (IM) carries not only conventional signal constellations as with conventional OFDM, but also secondary carrier indices.

In this research work a comparative analysis is performed among proposed softmax recurrent neural network based channel estimation with existing channel estimation technique for variable signal to noise ratio $(\mathrm{Eb} / \mathrm{No})$ from $1-30 \mathrm{db}$ for frequency indexed modulation techniques. From simulation result, it is observed and concluded that the existing channel estimation techniques gives higher BER as compared to proposed softmax recurrent neural network channel estimation technique. The proposed work is approx. $2 \%$ improvement of BER compared to previous result.

\section{REFERENCES}

[1] J. Liu, K. Mei, X. Zhang, D. Ma and J. Wei, "Online Extreme Learning Machine-Based Channel Estimation and Equalization for OFDM Systems," in IEEE Communications Letters, vol. 23, no. 7, pp. 12761279, July 2019.

[2] C. Liu, C. Chen, J. Lu and X. Dai, "A New Channel Estimation Algorithm for Time-Varying Multi-Path Channel in MIMO-OFDM Systems," 2019 IEEE 19th International Conference on Communication Technology (ICCT), Xi'an, China, 2019, pp. 69-74.

[3] Yujie Fan, Hui Li, Shuangshuang Song, Weisi Kong, Wenjie Zhang, "Structured Compressed Sensing-Based Time-Frequency Joint Channel Estimation For MIMO-OFDM Systems", IEEE Conference on Industrial Electronics and Applications (ICIEA), 2018, pp. 2006-2010. 
[4] Hao Wu, Member, Yuan Liu, and Kai Wang, "Analysis of DFT-Based Channel Estimation for Uplink Massive MIMO Systems", IEEE Communications Letters, Vol. 22, no. 2, 2018, pp. 328-331.

[5] Aqiel Almamori, Seshadri Mohan, "Estimation of Channel State Information for Massive MIMO Based on Received Data Using Kalman Filter" IEEE Computing and Communication Workshop and Conference (CCWC), 2018, pp. 665-669.

[6] Pham Hong Lien, Nguyen Due Quang and Luu Thanh Tra, "Extended Kalman Filter for Channel and Carrier Frequency Offset Estimation", International Conference on System Science and Engineering, 2017, pp. 61-65.

[7] Vincent Savaux, Yves Louët, "LMMSE channel estimation in OFDM context: a review", IET Signal Processing, Volume: 11, Issue: 2, 2017, pp. $123-134$.

[8] Ali Farzamnia, Ngu War Hlaing, Manas Kumar Haldar, Javad Rahebi, "Channel Estimation for Sparse Channel OFDM Systems using Least Square and Minimum Mean Square Error Techniques" ICET, IEEE, 2017.

[9] M.Raju and K.Ashoka Reddy, "Mean Square Error Analysis in MIMOOFDM System using Pilot based Channel Estimation”, International Conference on Signal Processing, Communication, Power and Embedded System (SCOPES), 2016, pp. 1631-1636.

[10] Kalpesh Hiray, K. Vinoth Babu, "A Neural Network Based Channel Estimation Scheme for OFDM System", International Conference on Communication and Signal Processing, 2016, pp. 438-441.

[11] V.B. Niranjane, N.A. Pande and A.A. Keluskar, "Adaptive Channel Estimation technique for MIMO-OFDM," International Journal of Emerging Technology and Advanced Engineering, vol. 3, Issue 1, pp. 692-696. 2013

[12] G. Gowri, G. Uma Maheswari, E. Vishnupriya, S. Prabha, D. Meenakshi, N. R. Raajan, "Performance Analysis of DWT-OFDM and FFT-OFDM Systems", International Journal of Engineering and Technology (IJET), Vol 5 No 2, pp.1455-1461, 2013.

[13] Yihua Yu and Yuan Liang, "Joint Carrier Frequency Offset and Channel Estimation for MIMO-OFDM Systems Using Extended $\mathrm{H}_{\infty}$ Filter", IEEE Communications Letters, vol. 16, no. 4, 2012, pp. 476478.

[14] A. Khlifi and R.Bouallegue, "Performance Analysis of LS and LMMSE Channel Estimation Techniques for LTE Downlink Systems". International Journal of Wireless \& Mobile Networks (IJWMN) vol. 3, no. 5, pp.141-149, 2011.

[15] Jun-Han Oh and Jong-Tae Lim, "Two-Step Channel Estimation Scheme for OFDM Systems over Fast Rayleigh Fading Channels", IEEE Communications Letters, vol. 14, no. 6, 2010, pp. 545-547. 